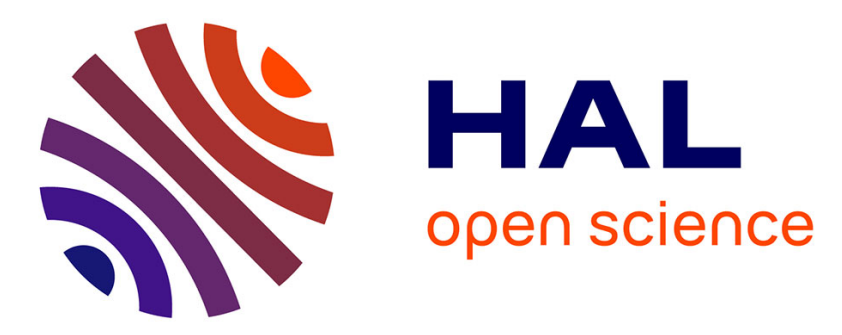

\title{
The Missing Difference Problem, and its Applications to Counter Mode Encryption
}

\author{
Gaëtan Leurent, Ferdinand Sibleyras
}

\section{To cite this version:}

Gaëtan Leurent, Ferdinand Sibleyras. The Missing Difference Problem, and its Applications to Counter Mode Encryption. JC2 2018 - Journées Codage et Cryptographie, Oct 2018, Aussois, France. hal-01961739

\section{HAL Id: hal-01961739 \\ https://inria.hal.science/hal-01961739}

Submitted on 20 Dec 2018

HAL is a multi-disciplinary open access archive for the deposit and dissemination of scientific research documents, whether they are published or not. The documents may come from teaching and research institutions in France or abroad, or from public or private research centers.
L'archive ouverte pluridisciplinaire HAL, est destinée au dépôt et à la diffusion de documents scientifiques de niveau recherche, publiés ou non, émanant des établissements d'enseignement et de recherche français ou étrangers, des laboratoires publics ou privés. 


\section{The Missing Difference Problem, and its Applications to Counter Mode Encryption}

Gaëtan Leurent, Ferdinand Sibleyras

Inria, équipe SECRET

Journées Codage \& Cryptographie 2018
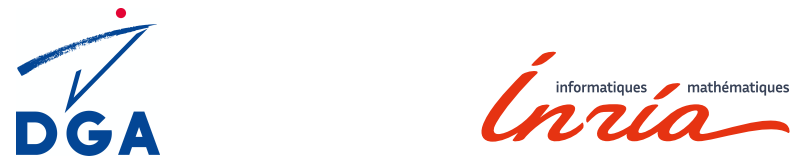


\section{Introduction}

- Cryptography: Alice encrypts then sends messages to Bob.

- Symmetric: Alice and Bob share the same key.

- Public channel: Eve (attacker) can see and/or manipulate what is being sent.

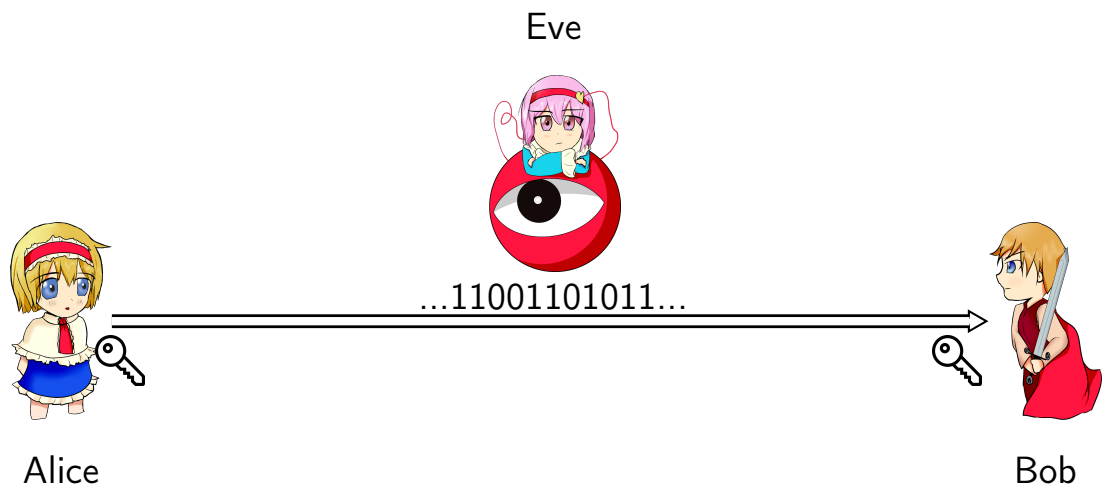




\section{Introduction}

\section{Block Cipher}

$$
E_{k}:\{0,1\}^{n} \rightarrow\{0,1\}^{n}
$$

A family of permutations indexed by a key (AES, 3DES, ...) where $n$ is the bit size of the permutation or block's size. 


\section{Introduction}

\section{Block Cipher}

$$
E_{k}:\{0,1\}^{n} \rightarrow\{0,1\}^{n}
$$

A family of permutations indexed by a key (AES, 3DES, ...) where $n$ is the bit size of the permutation or block's size.

Mode of operation

Describes how to use a block cipher along with a plaintext message of arbitrary length to achieve some concrete cryptographic goals. 


\section{The counter mode (CTR)}
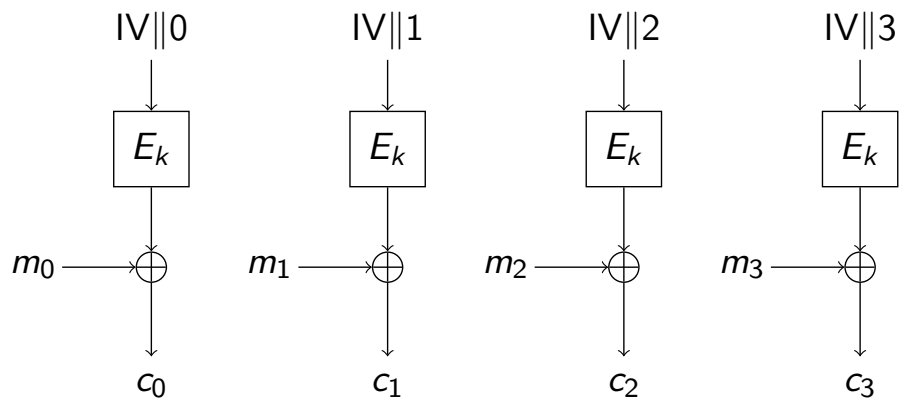

$m_{i}$ : The plaintext.

$E_{k}$ : The block cipher.

$c_{i}$ : The ciphertext.

IV : The Initialisation Value.

$$
c_{i}=E_{k}(\mathrm{IV} \| i) \oplus m_{i}
$$




\section{The counter mode (CTR)}
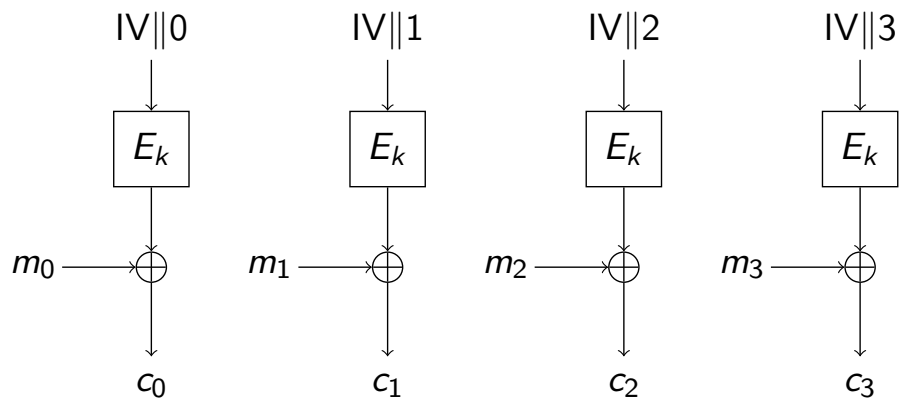

$m_{i}:$ The plaintext.

$E_{k}:$ The block cipher.

$c_{i}:$ The ciphertext.

IV : The Initialisation Value.

$$
c_{i}=E_{k}(\mathrm{IV} \| i) \oplus m_{i}
$$

Akin to a stream cipher: keystream XORed with the plaintext. 


\section{The counter mode (CTR)}
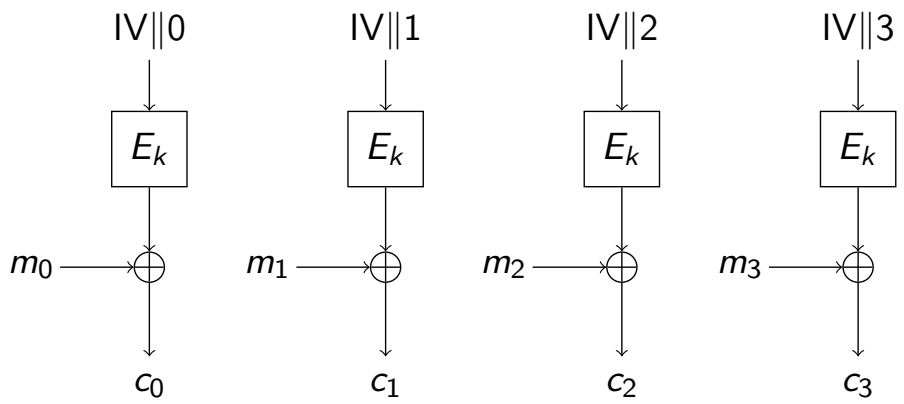

$m_{i}:$ The plaintext.

$E_{k}:$ The block cipher.

$c_{i}:$ The ciphertext.

IV : The Initialisation Value.

$$
c_{i}=E_{k}(\mathrm{IV} \| i) \oplus m_{i}
$$

Akin to a stream cipher: keystream XORed with the plaintext. Inputs IV $\| i$ to the block cipher never repeat. 


\section{The counter mode (CTR)}

Let $\quad K_{i}=E_{k}(\mathrm{IV} \| i) \quad$ the $i$ th block of keystream.

- If $E_{k}$ is a good Pseudo-Random Function (PRF) then all $K_{i}$ are random and this is a one-time-pad.

- A block cipher is a Pseudo-Random Permutation (PRP) therefore $K_{i}$ are all distinct: $K_{i} \neq K_{j} \forall i \neq j$. 


\section{The counter mode (CTR)}

Let $\quad K_{i}=E_{k}(\mathrm{IV} \| i) \quad$ the $i$ th block of keystream.

- If $E_{k}$ is a good Pseudo-Random Function (PRF) then all $K_{i}$ are random and this is a one-time-pad.

- A block cipher is a Pseudo-Random Permutation (PRP) therefore $K_{i}$ are all distinct: $K_{i} \neq K_{j} \forall i \neq j$.

\section{Security proof ( $\sigma$ the number of blocks)}

$\operatorname{Adv}_{\text {CTR- } E_{k}}^{\text {IND }}(\sigma) \leq \operatorname{Adv}_{E_{k}}^{\text {PRF }}(\sigma) \leq \operatorname{Adv}_{E_{k}}^{\text {PRP }}(\sigma)+\sigma^{2} / 2^{n+1}$

\section{Distinguisher}

After $\sigma \simeq 2^{n / 2}$ encrypted blocks we expect a collision on the $K_{i}$ with high probability in the case of a random ciphertext.

That is the birthday bound coming from the birthday paradox. 


\section{CBC and CTR}

Both modes are:

- widely deployed

- proven secure up to birthday bound $\left(2^{n / 2}\right)$

- matching distinguishers at the proof's bound

\section{CBC mode}

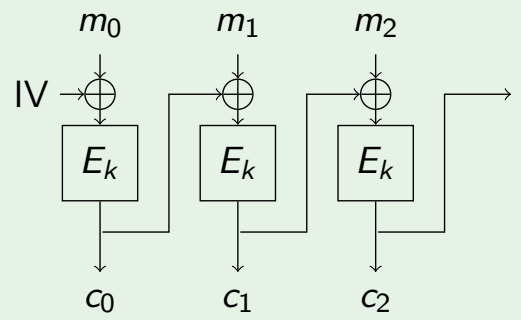




\section{CBC and CTR}

Both modes are:

- widely deployed

- proven secure up to birthday bound $\left(2^{n / 2}\right)$

- matching distinguishers at the proof's bound

\section{CBC mode}

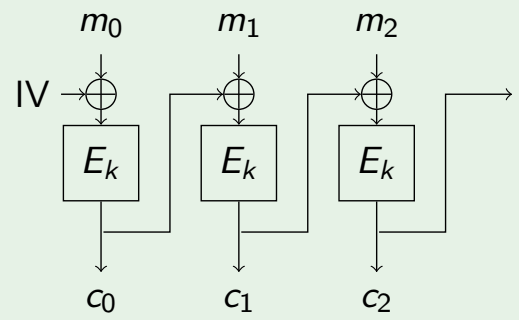

Folklore assumptions

[Ferguson, Schneier, Kohno]

CTR leaks very little data. [...] It would be reasonable to limit the cipher mode to $2^{60}$ blocks, which allows you to encrypt $2^{64}$ bytes but restricts the leakage to a small fraction of a bit.

When using CBC mode you should be a bit more restrictive. [...] We suggest limiting CBC encryption to $2^{32}$ blocks or so. 


\section{The counter mode (CTR)}

From a distinguishing attack to a plaintext recovery attack ?

- If we know $m_{i}$, we recover $K_{i}=c_{i} \oplus m_{i}$. 


\section{The counter mode (CTR)}

From a distinguishing attack to a plaintext recovery attack ?

- If we know $m_{i}$, we recover $K_{i}=c_{i} \oplus m_{i}$.

- We can observe repeated encryptions of a secret $S$ that is $c_{j}=K_{j} \oplus S$ for many different $j$. 


\section{The counter mode (CTR)}

From a distinguishing attack to a plaintext recovery attack ?

- If we know $m_{i}$, we recover $K_{i}=c_{i} \oplus m_{i}$.

- We can observe repeated encryptions of a secret $S$ that is $c_{j}=K_{j} \oplus S$ for many different $j$.

- The distinguisher uses $K_{i} \oplus K_{j} \neq 0$ which implies $K_{i} \oplus c_{j} \neq S \forall i \neq j$. 


\section{The counter mode (CTR)}

From a distinguishing attack to a plaintext recovery attack ?

- If we know $m_{i}$, we recover $K_{i}=c_{i} \oplus m_{i}$.

- We can observe repeated encryptions of a secret $S$ that is $c_{j}=K_{j} \oplus S$ for many different $j$.

- The distinguisher uses $K_{i} \oplus K_{j} \neq 0$ which implies $K_{i} \oplus c_{j} \neq S \forall i \neq j$.

\section{Main Idea}

Collect many keystream blocks $K_{i}$ and encryptions of secret block $c_{j}=K_{j} \oplus S$; then look for a value $S$ such that $K_{i} \oplus c_{j} \neq S \forall i \neq j$. 


\section{Missing difference problem}

The missing difference problem

- Given $\mathcal{A}$ and $\mathcal{B}$, and a hint $\mathcal{S}$ three sets of $n$-bit words

- Find $S \in \mathcal{S}$ such that:

$$
\forall(a, b) \in \mathcal{A} \times \mathcal{B}, \quad S \neq a \oplus b .
$$




\section{Missing difference problem}

\section{Main Idea}

Collect many keystream blocks $K_{i} \in \mathcal{A}$ and encryptions of secret block $c_{j}=K_{j} \oplus S \in \mathcal{B}$; then look for a value $S \in \mathcal{S}$ such that $\forall(a, b) \in \mathcal{A} \times \mathcal{B}, S \neq a \oplus b$.

The missing difference problem

- Given $\mathcal{A}$ and $\mathcal{B}$, and a hint $\mathcal{S}$ three sets of $n$-bit words

- Find $S \in \mathcal{S}$ such that:

$$
\forall(a, b) \in \mathcal{A} \times \mathcal{B}, \quad S \neq a \oplus b .
$$




\section{Simple Sieving Algorithm [McGrew, FSE'13]}

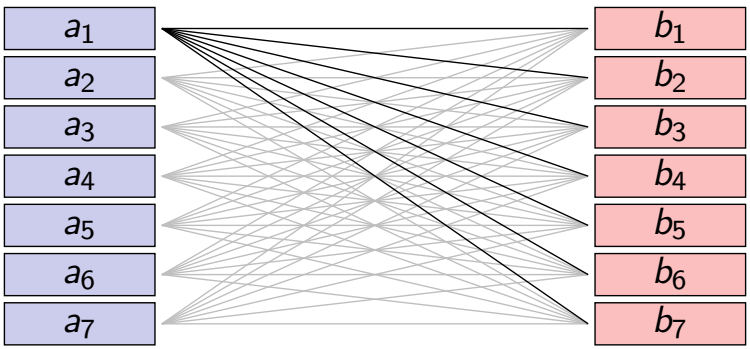

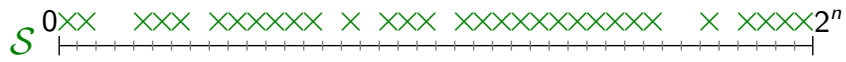

Compute all $a_{i} \oplus b_{j}$, remove results from a sieve $\mathcal{S}$.

Analysis: case $|\mathcal{S}|=2^{n}$ via coupon collector problem

- To exclude $2^{n}$ candidates of $S$, we need $n \cdot 2^{n}$ values $a_{i} \oplus b_{j}$

- Lists $\mathcal{A}$ and $\mathcal{B}$ of size $\sqrt{n} \cdot 2^{n / 2}$. Complexity: $\tilde{\mathcal{O}}\left(2^{n}\right)$ 


\section{Simple Sieving Algorithm [McGrew, FSE'13]}

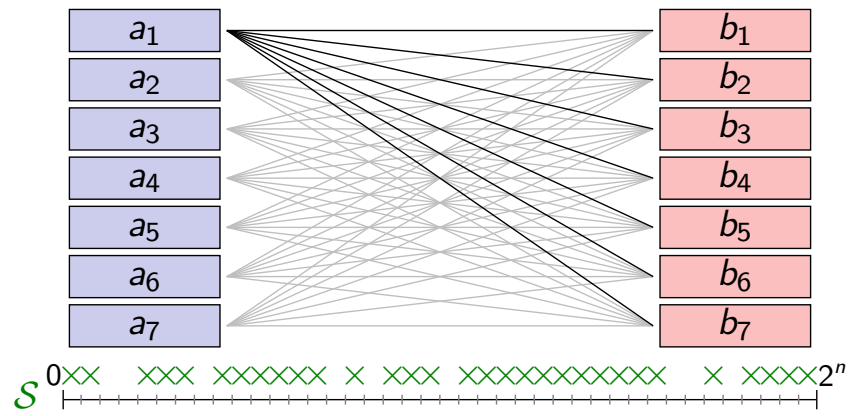

Compute all $a_{i} \oplus b_{j}$, remove results from a sieve $\mathcal{S}$.

Analysis: case $|\mathcal{S}|=2$

- To exclude 1 candidate of $S$, we need $2^{n}$ values $a_{i} \oplus b_{j}$

- Lists $\mathcal{A}$ and $\mathcal{B}$ of size $2^{n / 2}$. Complexity: $\tilde{\mathcal{O}}\left(2^{n}\right)$ 


\section{Searching Algorithm}

\section{[McGrew, FSE'13]}

\begin{tabular}{|l|}
\hline$a_{1}$ \\
\hline$a_{2}$ \\
\hline$a_{3}$ \\
\hline$a_{4}$ \\
\hline$a_{5}$ \\
\hline$a_{6}$ \\
\hline$a_{7}$ \\
\hline
\end{tabular}
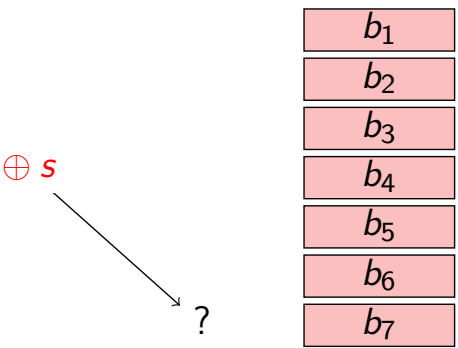

- Make a guess and verify.

Try Guess (s)

\section{for $a$ in $\mathcal{A}$ do}

$$
\begin{aligned}
& \text { if }(s \oplus a) \in \mathcal{B} \text { then } \\
& \text { return } 0
\end{aligned}
$$

return 1 


\section{Searching Algorithm}

[McGrew, FSE'13]

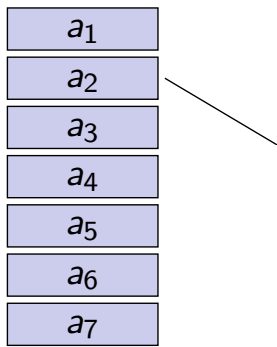

- Make a guess and verify.

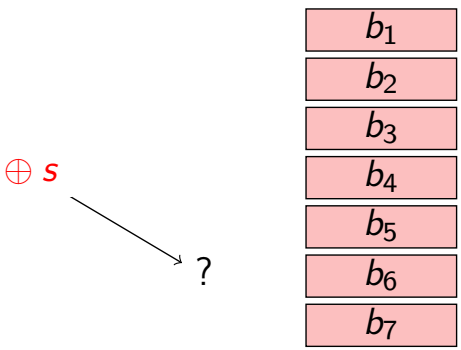

Try Guess (s) for $a$ in $\mathcal{A}$ do

$$
\begin{aligned}
& \text { if }(s \oplus a) \in \mathcal{B} \text { then } \\
& \text { return } 0
\end{aligned}
$$

return 1 


\section{Searching Algorithm}

[McGrew, FSE'13]

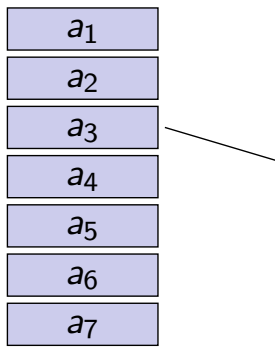

- Make a guess and verify.

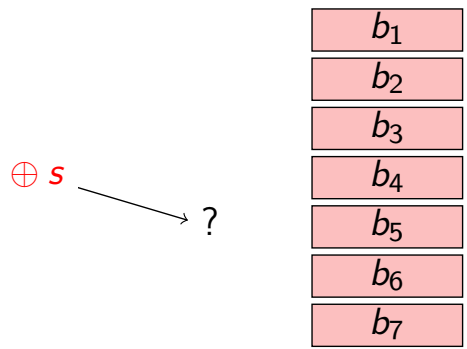

Try Guess ( $s$ ) for $a$ in $\mathcal{A}$ do

$$
\begin{aligned}
& \text { if }(s \oplus a) \in \mathcal{B} \text { then } \\
& \text { return } 0
\end{aligned}
$$

return 1 


\section{Searching Algorithm}

[McGrew, FSE'13]

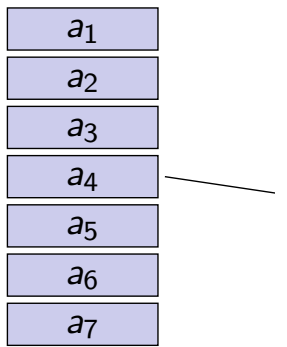

- Make a guess and verify. $\oplus s$

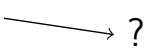

Try Guess ( $s$ )

$$
\text { if }(s \oplus a) \in \mathcal{B} \text { then }
$$
return 0

return 1

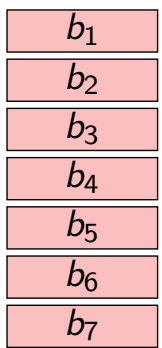




\section{Searching Algorithm}

[McGrew, FSE'13]

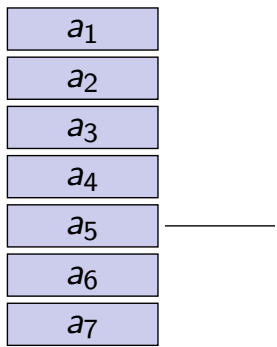

- Make a guess and verify.

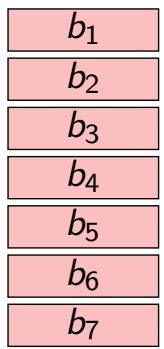

Try Guess (s)

for $a$ in $\mathcal{A}$ do

$$
\begin{aligned}
& \text { if }(s \oplus a) \in \mathcal{B} \text { then } \\
& \text { return } 0
\end{aligned}
$$

return 1 


\section{Searching Algorithm}

[McGrew, FSE'13]
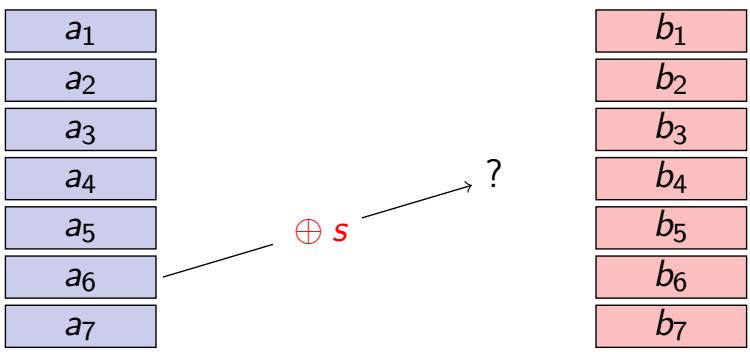

- Make a guess and verify.

Try Guess (s)

for $a$ in $\mathcal{A}$ do

$$
\begin{aligned}
& \text { if }(s \oplus a) \in \mathcal{B} \text { then } \\
& \text { return } 0
\end{aligned}
$$

return 1 


\section{Searching Algorithm}

[McGrew, FSE'13]

\begin{tabular}{|l|}
\hline$a_{1}$ \\
\hline$a_{2}$ \\
\hline$a_{3}$ \\
\hline$a_{4}$ \\
\hline$a_{5}$ \\
\hline$a_{6}$ \\
\hline$a_{7}$ \\
\hline
\end{tabular}

- Make a guess and verify.

- Complexity $\tilde{\mathcal{O}}\left(2^{n / 2} \sqrt{|\mathcal{S}|}\right)$ with unbalanced $\mathcal{A}, \mathcal{B}$.

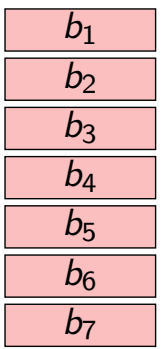

Try Guess (s)

for $a$ in $\mathcal{A}$ do

$$
\begin{aligned}
& \text { if }(s \oplus a) \in \mathcal{B} \text { then } \\
& \text { return } 0
\end{aligned}
$$

return 1 


\section{Known-prefix Sieving}

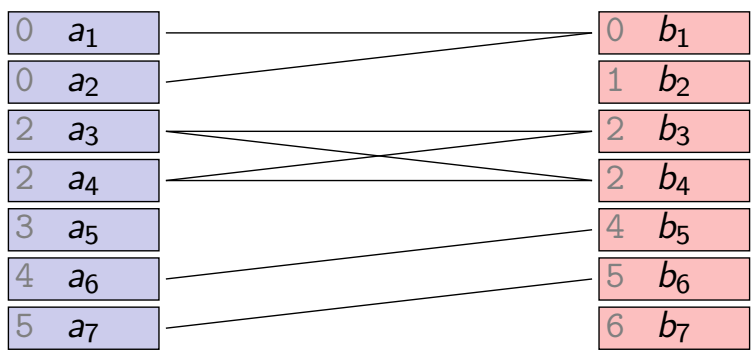

$\mathcal{S} \stackrel{0 \times X X \times X \times X \times 2^{n-z}}{1}$

- Assume $S$ starts with z zero bits (more generally, linear subspace with $\operatorname{dim}\langle\mathcal{S}\rangle=n-z$ )

- Sort lists, consider $a_{i}$ 's and $b_{j}$ 's with matching z-bit prefix

- Complexity: $\tilde{\mathcal{O}}\left(2^{n / 2}+2^{\operatorname{dim}\langle\mathcal{S}\rangle}\right)$

- Looking for collision + needed number of collisions

- Complexity: $\tilde{\mathcal{O}}\left(2^{n / 2}\right)$ when $\operatorname{dim}\langle\mathcal{S}\rangle \leq n / 2$ 


\section{Fast Convolution Sieving}
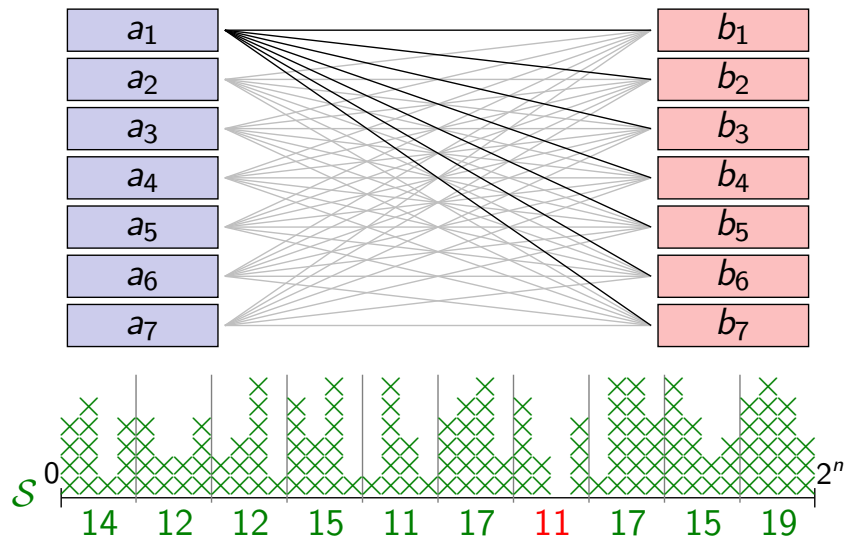

- Instead of computing full sieve, use buckets (ie. truncate)

- With enough data, missing difference has smallest bucket with high probability 


\section{Computing the sieve}

- Count buckets for $\mathcal{A}$ and $\mathcal{B}$

- $C_{\mathcal{X}}[i]=|\{x \in \mathcal{X} \mid T(x)=i\}|$
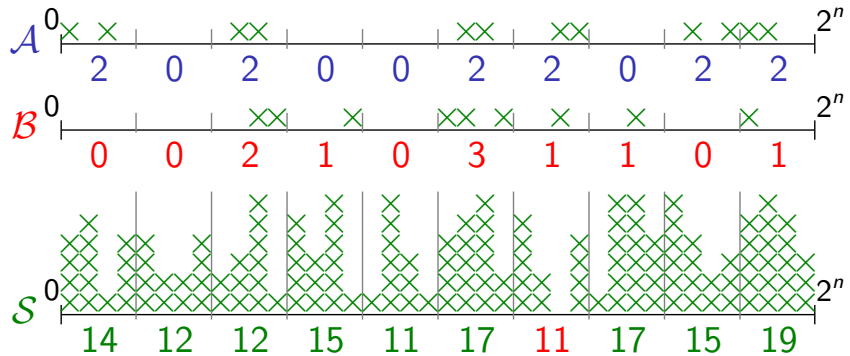


\section{Computing the sieve}

- Count buckets for $\mathcal{A}$ and $\mathcal{B}$

- $C_{\mathcal{X}}[i]=|\{x \in \mathcal{X} \mid T(x)=i\}|$

- $C_{\mathcal{S}}[i]=|\{(a, b) \in \mathcal{A} \times \mathcal{B} \mid T(a \oplus b)=i\}|$

$=\sum_{a \in \mathcal{A}}|\{b \in \mathcal{B} \mid T(a \oplus b)=i\}|$

$=\sum_{a \in \mathcal{A}} C_{\mathcal{B}}[i \oplus T(a)]$

$=\sum C_{\mathcal{A}}[j] \cdot C_{\mathcal{B}}[i \oplus j]$

$j \in\{0,1\}^{n-t}$ 


\section{Computing the sieve}

- Count buckets for $\mathcal{A}$ and $\mathcal{B}$

- $C_{\mathcal{X}}[i]=|\{x \in \mathcal{X} \mid T(x)=i\}|$

- $C_{\mathcal{S}}[i]=|\{(a, b) \in \mathcal{A} \times \mathcal{B} \mid T(a \oplus b)=i\}|$

$=\sum_{a \in \mathcal{A}}|\{b \in \mathcal{B} \mid T(a \oplus b)=i\}|$

$=\sum_{a \in \mathcal{A}} C_{\mathcal{B}}[i \oplus T(a)]$

$=\sum_{j \in\{0,1\}^{n-t}} C_{\mathcal{A}}[j] \cdot C_{\mathcal{B}}[i \oplus j]$

- Discrete convolution can be computed efficiently with the Fast Walsh-Hadamard transform!

- Complexity: $\tilde{\mathcal{O}}\left(\left|C_{\mathcal{S}}\right|\right)$ for arbitrary $\mathcal{S}$ 


\section{Fast Convolution Sieving}
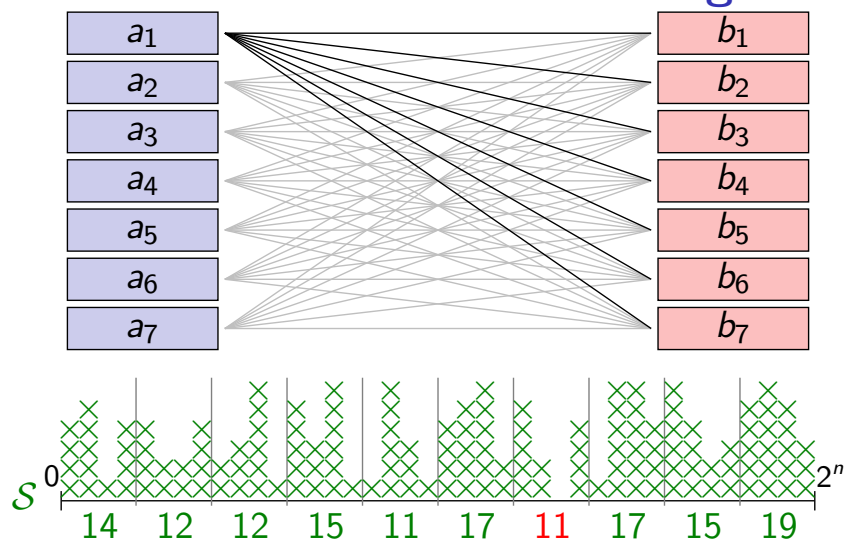

$$
T(S) \stackrel{?}{=} \operatorname{argmin} C_{\mathcal{S}}[i]
$$

And we can finish with Known-prefix Sieving to recover the rest.

- $2^{2 n / 3}$ queries, sieving with $2^{2 n / 3}$ buckets of $2^{n / 3}$ elements 


\section{Missing difference problem algorithms}

Algorithms for the missing difference problem

$$
\begin{array}{cc}
\text { Simple Sieving Complexity } \tilde{\mathcal{O}}\left(2^{n}\right) & \text { [McGrew] } \\
\text { Searching Complexity } \tilde{\mathcal{O}}\left(2^{n / 2} \sqrt{|\mathcal{S}|}\right) & {[\text { McGrew] }}
\end{array}
$$

Known-prefix Sieving Complexity $\tilde{\mathcal{O}}\left(2^{n / 2}+2^{\operatorname{dim}\langle\mathcal{S}\rangle}\right)$

Fast Convolution Sieving Complexity $\tilde{\mathcal{O}}\left(2^{2 n / 3}\right)$ 


\section{Missing difference problem algorithms}

\section{Algorithms for the missing difference problem}

$$
\text { Simple Sieving Complexity } \tilde{\mathcal{O}}\left(2^{n}\right)
$$

Searching Complexity $\tilde{\mathcal{O}}\left(2^{n / 2} \sqrt{|\mathcal{S}|}\right)$

[McGrew]

Known-prefix Sieving Complexity $\tilde{\mathcal{O}}\left(2^{n / 2}+2^{\operatorname{dim}\langle\mathcal{S}\rangle}\right)$

Fast Convolution Sieving Complexity $\tilde{\mathcal{O}}\left(2^{2 n / 3}\right)$

- Improved algorithm if $\mathcal{S}$ is a linear subspace

- In particular still near optimal when $\operatorname{dim}\langle\mathcal{S}\rangle=n / 2$

- Improved algorithm for arbitrary $\mathcal{S}$ at the cost of data

- First algorithm with complexity below $2^{n}$ in that case 


\section{Back to Cryptanalysis}

New Tools, New Attacks

Known-prefix $\rightarrow$ plaintext recovery on CTR mode

Fast Convolution $\rightarrow$ forgery on GMAC and Poly1305 


\section{BEAST Attack Setting [Duong \& Rizzo 2011]}

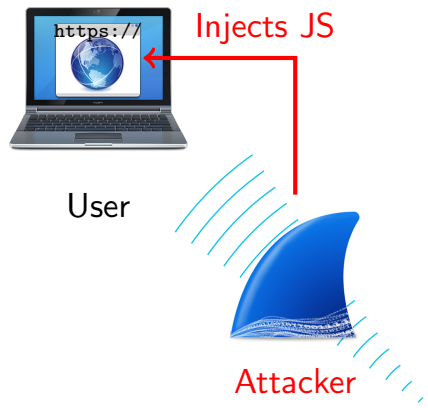

- Attacker has access to the network (eg. public WiFi)

1. Attacker uses JS to generate traffic

- Tricks victim to malicious site

- JS makes cross-origin requests

2. Attacker captures encrypted data

- Chosen plaintext attack

- Chosen-Prefix Secret-Suffix model $M \rightarrow \mathcal{E}(M \| S)$

Captures

encrypted traffic

[Hoang \&al., Crypto'15]

Public WiFi 


\section{Application to CTR (CPSS queries)}

- Plaintext recovery using the known-prefix sieving algorithm

- Two kind of queries; half-block and full-block headers:

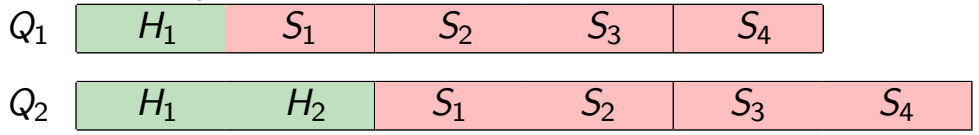

1. Recover $S_{1}$ using the first block of each query:

$$
\left.\begin{array}{l}
\mathcal{A}=\left\{\mathcal{E}\left(H_{1} \| H_{2}\right)\right\} \\
\mathcal{B}=\left\{\mathcal{E}\left(H_{1} \| S_{1}\right)\right\}
\end{array}\right\} \rightarrow \text { Missing difference: } \quad 0 \|\left(S_{1} \oplus H_{2}\right)
$$




\section{Application to CTR (CPSS queries)}

- Plaintext recovery using the known-prefix sieving algorithm

- Two kind of queries; half-block and full-block headers:

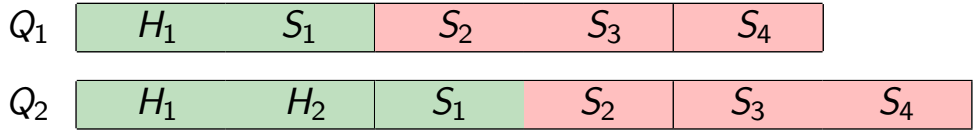

1. Recover $S_{1}$ using the first block of each query:

$$
\left.\begin{array}{l}
\mathcal{A}=\left\{\mathcal{E}\left(H_{1} \| H_{2}\right)\right\} \\
\mathcal{B}=\left\{\mathcal{E}\left(H_{1} \| S_{1}\right)\right\}
\end{array}\right\} \rightarrow \text { Missing difference: } \quad 0 \|\left(S_{1} \oplus H_{2}\right)
$$

2. When $S_{1}$ is known, recover $S_{2}$, with $Q_{2}$ queries:

$$
\left.\begin{array}{l}
\mathcal{A}=\left\{\mathcal{E}\left(H_{1} \| H_{2}\right)\right\} \\
\mathcal{B}=\left\{\mathcal{E}\left(S_{1} \| S_{2}\right)\right\}
\end{array}\right\} \rightarrow \text { Missing difference: }\left(S_{1} \oplus H_{1}\right) \|\left(S_{2} \oplus H_{2}\right)
$$




\section{Application to CTR (CPSS queries)}

- Plaintext recovery using the known-prefix sieving algorithm

- Two kind of queries; half-block and full-block headers:

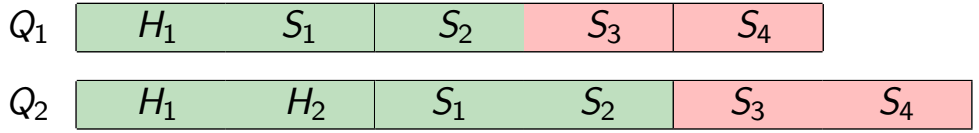

1. Recover $S_{1}$ using the first block of each query:

$$
\left.\begin{array}{l}
\mathcal{A}=\left\{\mathcal{E}\left(H_{1} \| H_{2}\right)\right\} \\
\mathcal{B}=\left\{\mathcal{E}\left(H_{1} \| S_{1}\right)\right\}
\end{array}\right\} \rightarrow \text { Missing difference: } \quad 0 \|\left(S_{1} \oplus H_{2}\right)
$$

2. When $S_{1}$ is known, recover $S_{2}$, with $Q_{2}$ queries:

$$
\left.\begin{array}{l}
\mathcal{A}=\left\{\mathcal{E}\left(H_{1} \| H_{2}\right)\right\} \\
\mathcal{B}=\left\{\mathcal{E}\left(S_{1} \| S_{2}\right)\right\}
\end{array}\right\} \rightarrow \text { Missing difference: }\left(S_{1} \oplus H_{1}\right) \|\left(S_{2} \oplus H_{2}\right)
$$

3. When $S_{2}$ is known, recover $S_{3}$ :

$$
\left.\begin{array}{l}
\mathcal{A}=\left\{\mathcal{E}\left(H_{1} \| H_{2}\right)\right\} \\
\mathcal{B}=\left\{\mathcal{E}\left(S_{2} \| S_{3}\right)\right\}
\end{array}\right\} \rightarrow \text { Missing difference: }\left(S_{2} \oplus H_{1}\right) \|\left(S_{3} \oplus H_{2}\right) \text {. }
$$

4. ... 


\section{Application to CTR (CPSS queries)}

Full Asymptotic Complexity
Queries $\mathcal{O}\left(\sqrt{n} \cdot 2^{n / 2}\right)$
Memory $\mathcal{O}\left(\sqrt{n} \cdot 2^{n / 2}\right)$
Time $\mathcal{O}\left(n \cdot 2^{n / 2}\right)$ 


\section{Impacts}

How practical can be the plaintext recovery attack on CTR ?

- Mostly used with AES, famous 128-bit block cipher, as part of GCM. $90 \%$ of Firefox HTTPS traffic uses AES-GCM.

- Requires $128 \times 2^{64}$ bits $=256$ exbibytes over one session

- 2016 global IP traffic is 82.3 exbibytes per month [Cisco] 


\section{Impacts}

How practical can be the plaintext recovery attack on CTR ?

- Mostly used with AES, famous 128-bit block cipher, as part of GCM. $90 \%$ of Firefox HTTPS traffic uses AES-GCM.

- Requires $128 \times 2^{64}$ bits $=256$ exbibytes over one session

- 2016 global IP traffic is 82.3 exbibytes per month [Cisco]

- SSHv2 includes CTR with 3DES, a 64-bit block cipher.

- Requires $64 \times 2^{32}$ bits $=32 \mathrm{GiB}$

- Quickly attainable with modern internet speed 


\section{Impacts}

How practical can be the plaintext recovery attack on CTR ?

- Mostly used with AES, famous 128-bit block cipher, as part of GCM. $90 \%$ of Firefox HTTPS traffic uses AES-GCM.

- Requires $128 \times 2^{64}$ bits $=256$ exbibytes over one session

- 2016 global IP traffic is 82.3 exbibytes per month [Cisco]

- SSHv2 includes CTR with 3DES, a 64-bit block cipher.

- Requires $64 \times 2^{32}$ bits $=32 \mathrm{GiB}$

- Quickly attainable with modern internet speed

\section{Sweet32 attack by Bhargavan and Leurent}

Attack in the BEAST setting with birthday bound complexity already shown to be a threat over the web in recent work. This is the Sweet32 attack on CBC mode, more commonly used with 64-bit block ciphers. 


\section{Wegman-Carter Authentication Modes}

- Wegman-Carter: build a MAC from a universal hash function and a PRF

$$
\begin{aligned}
& \operatorname{WC}(N, M)=H_{k_{1}}(M) \oplus F_{k_{2}}(N) . \\
& \operatorname{Adv}_{\mathrm{WC}[H, F]}^{\mathrm{MAC}} \leq \operatorname{Adv}_{F}^{\mathrm{PRF}}+\varepsilon+2^{-n}
\end{aligned}
$$

- Wegman-Carter-Shoup: use a block cipher as a PRF

$$
\operatorname{WCS}(N, M)=H_{k_{1}}(M) \oplus E_{k_{2}}(N) \text {, }
$$

Example: Polynomial-based hashing (GMAC, Poly1305-AES)

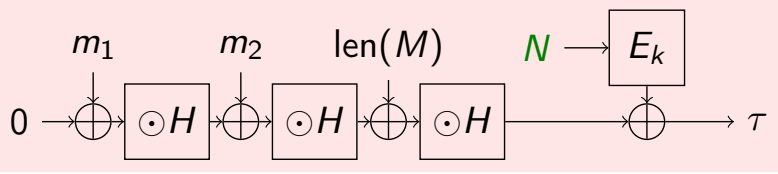




\section{Key recovery as a missing difference problem}

- Fix two messages $M \neq M^{\prime}$, capture MACs

- $a_{\mathrm{i}}=\operatorname{MAC}(\mathrm{i}, M)=H_{K_{\mathbf{1}}}(M) \oplus K_{i}$

- $b_{j}=\operatorname{MAC}\left(j, M^{\prime}\right)=H_{K_{1}}\left(M^{\prime}\right) \oplus K_{j}$

- $a_{i} \oplus b_{j} \neq H_{K_{1}}(M) \oplus H_{K_{1}}\left(M^{\prime}\right)$

- For polynomial hashing, easy to recover universal hash key from $H_{K_{1}}(M) \oplus H_{K_{1}}\left(M^{\prime}\right)$ 


\section{Key recovery as a missing difference problem}

- Fix two messages $M \neq M^{\prime}$, capture MACs

- $a_{\mathrm{i}}=\operatorname{MAC}(\mathrm{i}, M)=H_{K_{\mathbf{1}}}(M) \oplus K_{i}$

- $b_{j}=\operatorname{MAC}\left(j, M^{\prime}\right)=H_{K_{1}}\left(M^{\prime}\right) \oplus K_{j}$

- $a_{i} \oplus b_{j} \neq H_{K_{1}}(M) \oplus H_{K_{1}}\left(M^{\prime}\right)$

- For polynomial hashing, easy to recover universal hash key from $H_{K_{1}}(M) \oplus H_{K_{1}}\left(M^{\prime}\right)$

- Sieving algorithm recovers $H(M) \oplus H\left(M^{\prime}\right)$ with $\tilde{\mathcal{O}}\left(2^{n / 2}\right)$ queries and $\tilde{\mathcal{O}}\left(2^{n}\right)$ computations

- Independently done in another Eurocrypt paper!

Optimal Forgeries Against Polynomial-Based MACs and GCM Atul Luykx, Bart Preneel

[Eurocrypt '18] 


\section{Key recovery as a missing difference problem}

- Fix two messages $M \neq M^{\prime}$, capture MACs

- $a_{\mathrm{i}}=\operatorname{MAC}(\mathrm{i}, M)=H_{K_{\mathbf{1}}}(M) \oplus K_{i}$

- $b_{j}=\operatorname{MAC}\left(j, M^{\prime}\right)=H_{K_{1}}\left(M^{\prime}\right) \oplus K_{j}$

- $a_{i} \oplus b_{j} \neq H_{K_{1}}(M) \oplus H_{K_{1}}\left(M^{\prime}\right)$

- For polynomial hashing, easy to recover universal hash key from $H_{K_{1}}(M) \oplus H_{K_{1}}\left(M^{\prime}\right)$

- Sieving algorithm recovers $H(M) \oplus H\left(M^{\prime}\right)$ with $\tilde{\mathcal{O}}\left(2^{n / 2}\right)$ queries and $\tilde{\mathcal{O}}\left(2^{n}\right)$ computations

- Independently done in another Eurocrypt paper!

证 Optimal Forgeries Against Polynomial-Based MACs and GCM Atul Luykx, Bart Preneel

[Eurocrypt '18]

- Fast convolution sieving recovers $H(M) \oplus H\left(M^{\prime}\right)$ with $\tilde{\mathcal{O}}\left(2^{2 n / 3}\right)$ queries and computations

- First universal forgery attack with less than $2^{n}$ operations 


\section{Bonus algorithm}

\section{Citation}

[Luykx \& Preneel, Eurocrypt'18]

... implementing the attacks seems to require a large amount of storage to achieve significant success probability. It is unclear whether there is a compact way of representing the set of false keys. 


\section{Bonus algorithm}

\section{Citation}

[Luykx \& Preneel, Eurocrypt'18]

... implementing the attacks seems to require a large amount of storage to achieve significant success probability. It is unclear whether there is a compact way of representing the set of false keys.

Optimal queries and memory complete sieving

Guess first half of difference.

Run Known-prefix sieving over second half.

Repeat until found. 


\section{Bonus algorithm}

\section{Citation}

[Luykx \& Preneel, Eurocrypt'18]

... implementing the attacks seems to require a large amount of storage to achieve significant success probability. It is unclear whether there is a compact way of representing the set of false keys.

\section{Optimal queries and memory complete sieving}

Guess first half of difference.

Run Known-prefix sieving over second half.

Repeat until found.

Time is still $\tilde{\mathcal{O}}\left(2^{n}\right)$ but memory reduced to $\mathcal{O}\left(2^{n / 2}\right)$ in the nonce-respecting CPA model. 


\section{Conclusion}

We defined the missing difference problem and improved the algorithms to solve it in particular for some cases:

\begin{tabular}{|c|c|c|c|}
\hline Case & Previous & This work & Improved attacks \\
\hline $\begin{array}{c}\mathcal{S} \text { affine subspace } \\
\text { of } \operatorname{dim} n / 2\end{array}$ & $\tilde{\mathcal{O}}\left(2^{3 n / 4}\right)$ & $\tilde{\mathcal{O}}\left(2^{n / 2}\right)$ & $\begin{array}{c}\text { CTR } \\
\text { plaintext recovery }\end{array}$ \\
\hline $\begin{array}{c}\text { No prior info } \\
\text { ie. }|\mathcal{S}|=2^{n}\end{array}$ & $\tilde{\mathcal{O}}\left(2^{n}\right)$ & $\tilde{\mathcal{O}}\left(2^{2 n / 3}\right)$ & $\begin{array}{c}\text { GMAC, Poly1305 } \\
\text { universal forgery }\end{array}$ \\
\hline
\end{tabular}




\section{Conclusion}

We defined the missing difference problem and improved the algorithms to solve it in particular for some cases:

\begin{tabular}{|c|c|c|c|}
\hline Case & Previous & This work & Improved attacks \\
\hline $\begin{array}{c}\mathcal{S} \text { affine subspace } \\
\text { of } \operatorname{dim} n / 2\end{array}$ & $\tilde{\mathcal{O}}\left(2^{3 n / 4}\right)$ & $\tilde{\mathcal{O}}\left(2^{n / 2}\right)$ & $\begin{array}{c}\text { CTR } \\
\text { plaintext recovery }\end{array}$ \\
\hline $\begin{array}{c}\text { No prior info } \\
\text { ie. }|\mathcal{S}|=2^{n}\end{array}$ & $\tilde{\mathcal{O}}\left(2^{n}\right)$ & $\tilde{\mathcal{O}}\left(2^{2 n / 3}\right)$ & $\begin{array}{c}\text { GMAC, Poly1305 } \\
\text { universal forgery }\end{array}$ \\
\hline
\end{tabular}

Main take away :

- CTR mode not more secure than CBC (Sweet32).

- Frequent rekeying away from birthday bound will prevent these attacks. 


\section{Known-prefix Sieving Simulation}

We challenge the heuristic assumptions we made (independence of the XORs $\{a \oplus b\})$. Approximations seem good enough.

Ran simulations with $n=64$ bits and $z=n / 2=32$ zeros.

- Each round we compare two lists of $2^{n / 2}$ elements.

- Each round we expect $2^{n / 2}$ partial collisions.

- Coupon collector predicts $n / 2 \cdot \ln (2) \cdot 2^{n / 2}$ partial collisions to recover $S$, that is 23 rounds on expectation.

- Simulation gives an idea of what is hidden in the $\mathcal{O}$ notations.

Consistent speed of leaking

In every runs, after 16 rounds the sieve was left between 419 and 560 candidates of $S$ only. 


\section{Known-prefix Sieving Simulation}

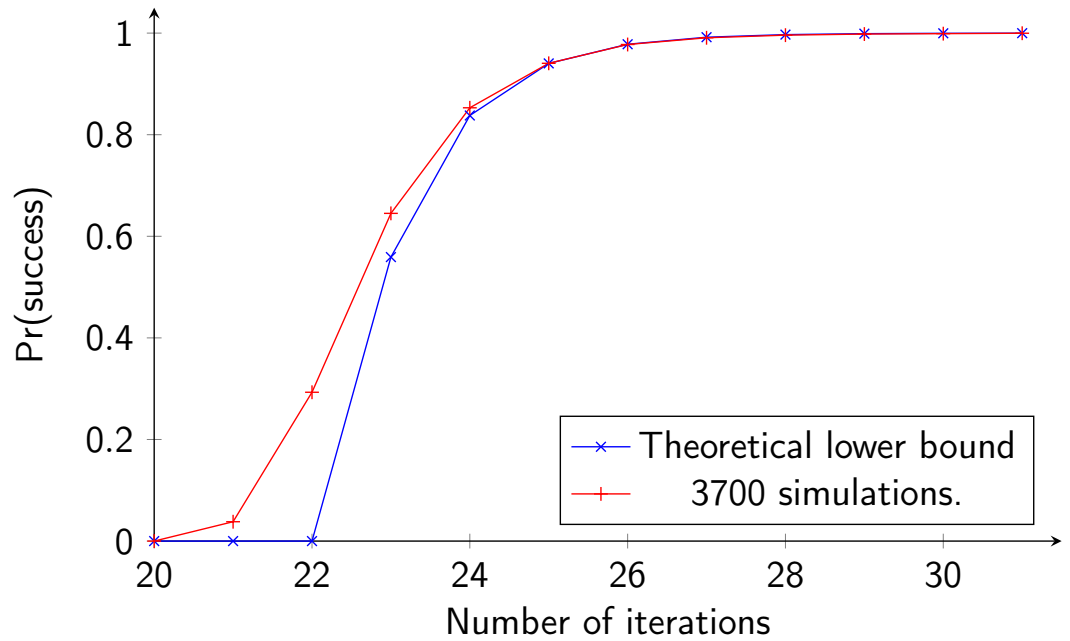

Figure: Probability of success of the known prefix sieving knowing $2^{32}$ encryptions of a 32-bit secret against the number of chunks of $2^{32}$ keystream blocks of size $n=64$ bits used. 


\section{Fast Convolution Simulation}

Figure: Results for $\sqrt{n} 2^{2 n / 3}$ data; counting over $2 n / 3$ bits.

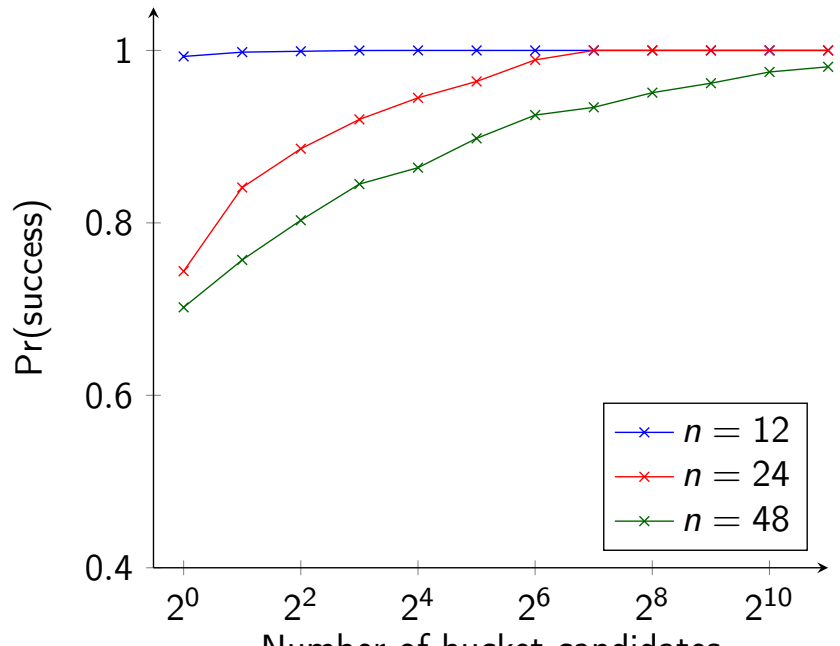

Number of bucket candidates 


\section{Works comparison}

We independently described roughly the same attack on GCM, yet luckily our works complete each others:

\section{Leurent \& Sibleyras, EC'18}

- Computational model

- Focus on algorithms

- Run simulations

- Provide a range of novel techniques and trade-offs

- Approach extendable to forgery on CWC mode

\section{Luykx \& Preneel, EC'18}

- Information theoretic model

- Focus on proofs

- More rigorous analysis

- Show optimality w.r.t the best proofs

- Approach extendable to the KPA setting 